\title{
Centrality and Multiparticle Production in Ultrarelativistic Nuclear Collisions
}

\author{
T. A. Drozhzhova, V. N. Kovalenko, A. Yu. Seryakov, and G. A. Feofilov* \\ St. Petersburg State University, Universitetskaya Naberezhnaya 7-9, St. Petersburg, 199034 Russia
}

Received December 21, 2015

\begin{abstract}
A critical analysis of methods for selecting central events in high-energy proton-nucleus $(p A)$ and nucleus-nucleus $(A A)$ collisions is presented. A sample of event classes in which background fluctuations associated with the dispersion of the impact parameter of each event or the number of participant nucleons are minimal is examined. At the SPS and LHC energies, the numbers of nucleonnucleon collisions are estimated with the aid of the Monte Carlo event generators HIJING and AMPT, which take into account energy-momentum conservation, and on the basis of a non-Glauber model involving string fusion and a modified Glauber model. The results obtained in this way demonstrate the need for revising the extensively used application of the Glauber model in normalizing multiplicity yields in experimental data on $p A$ and $A A$ collisions in the soft region of the spectrum.
\end{abstract}

DOI: $10.1134 / \mathrm{S} 1063778816040074$

\section{INTRODUCTION}

Hot and dense strongly interacting matter under extreme pressure and temperature conditions is produced in ultrarelativistic nucleus-nucleus collisions in the form of a quark-gluon plasma (QGP) and is characterized within thermodynamic models by quantities like the baryochemical potential, temperature, specific heat, and compressibility parameter [1$3]$. The fundamental properties of this matter are reflected in its global observables, including the multiplicity of product particles, their content, the average transverse momentum, and the residual electrical and baryon charges. Knowledge of initial conditions in high-energy nucleus-nucleus and proton-nucleus collisions is of importance for analyzing processes leading to the formation of quark-gluon plasma and its subsequent hadronization. A survey of theoretical approaches used to study and characterize initial states in ultrarelativistic nucleus-nucleus collisions can be found, for example, in [4, 5].

The need for quantitative estimations at early stages of hadron collisions became obvious upon an analysis of experimental data on multiparticle production processes in collisions of ultrarelativistic lead nuclei at the Large Hadron Collider (LHC at CERN). It turned out that, without respective estimates, it was impossible to study the properties of product quarkgluon plasma, such as viscosity and the entropy density. The role of initial conditions manifested itself most acutely even in the first results on $p \mathrm{~Pb}$ collisions

\footnotetext{
E-mail: grigory-feofilov@yandex.ru
}

at the LHC, where, in high-multiplicity events, the behavior of many observables proved to be close to the picture observed in $\mathrm{PbPb}$ collisions [6-13].

The conclusion that multiparticle production processes in nucleus-nucleus collisions differ substantially from what one expects in the case of a simple superposition of independent nucleon-nucleon collisions with a mean multiplicity of $N_{\text {coll }} d N / d \eta$, where $N_{\text {coll }}$ is the number of binary collisions and $d N / d \eta$ is the multiplicity in proton-proton $(p p)$ collisions at the same energy in the center-of-mass (c.m.) frame, was one of the important observations in experimental data on $\mathrm{AuAu}$ and $\mathrm{PbPb}$ collisions at the Relativistic Heavy-Ion Collider (RHIC) and at the LHC. In nucleus-nucleus $(A A)$ collisions, the experimentally measured mean multiplicity $\left\langle N_{\mathrm{ch}}\right\rangle$ of charged particles is substantially smaller than $N_{\text {coll }} d N / d \eta$. This fact may suggest either the presence of strong coherence effects or a manifestation of additional degrees of freedom at the subnucleon level [4]. In view of this, investigations into proton-nucleus $(p \mathrm{~Pb})$ collisions is an important part of the research program for the LHC, since they will provide additional physics information for interpreting data on nucleus-nucleus collisions. It is reasonable to assume that measurements for $p \mathrm{~Pb}$ and $p p$ collisions at the LHC and a comparative analysis of their results will make it possible to separate phenomena associated with QGP formation from effects of ordinary nuclear matter.

A comparison of transverse-momentum spectra measured in $\mathrm{PbPb}$ and $p \mathrm{~Pb}$ collisions with respective reference spectra in $p p$ collisions is one of the methods 
for such an analysis. This makes it possible to obtain extensively used values of the nuclear-modification factors. In the case of $p \mathrm{~Pb}$ collisions, these factors are denoted by $R_{p \mathrm{~Pb}}$ and are defined as [12-14]

$$
\begin{gathered}
R_{p \mathrm{~Pb}}\left(p_{T}\right)=\frac{d^{2} N_{\mathrm{ch}}^{p \mathrm{~Pb}} / d p_{T} d \eta}{\left\langle T_{p \mathrm{~Pb}}\right\rangle d^{2} \sigma_{\text {inel }}^{p p} / d p_{T} d \eta} \\
=\frac{d^{2} N_{\mathrm{ch}}^{p \mathrm{~Pb}} / d p_{T} d \eta}{\left\langle N_{\mathrm{coll}}\right\rangle d^{2} N_{\mathrm{ch}}^{p p} / d p_{T} d \eta} .
\end{gathered}
$$

In (1), $N_{\mathrm{ch}}^{p \mathrm{~Pb}}$ is the mean charged-particle-multiplicity yield in some class of centrality of $p \mathrm{~Pb}$ collisions, $\left\langle N_{\text {coll }}\right\rangle$ is the mean number of nucleon-nucleon collisions, $\left\langle T_{p \mathrm{~Pb}}\right\rangle=\left\langle N_{\text {coll }}\right\rangle / \sigma_{\text {inel }}^{p p}$ is the mean nuclearoverlap function for the same centrality class, and $\sigma_{\text {inel }}^{p p}$ is the cross section for inelastic nucleon-nucleon interaction.

One assumes that the nuclear-modification factor should be equal to unity for rare processes of hard parton collisions, and this may correspond to the case of an incoherent superposition of nucleon-nucleon collisions. In this approach, the deviation of $R_{p \mathrm{~Pb}}$ from unity may suggest either a manifestation of final-state effects associated with the parton energy loss via interaction with dense matter or a manifestation of initial-state effects \{for example, an in-medium modification of parton distributions (nPDF) [15] and shadowing [16], the Cronin effect [17], and gluondensity saturation [5] (for an overview of theoretical predictions for the LHC, see [18])\}.

Knowledge of centrality of nucleus-nucleus and proton-nucleus collisions (and respective values of $\left\langle N_{\text {part }}\right\rangle, N_{\text {ch }}^{p \mathrm{~Pb}},\left\langle N_{\text {coll }}\right\rangle$, and $\left.\left\langle T_{p \mathrm{~Pb}}\right\rangle\right)$ is of importance first of all in exploring and analyzing mean values of global observables that determine the properties of QCD matter and which are sensitive to temperature and density values.

In addition, it is worth noting that multiparticleproduction processes in hadron collisions have a statistical character, and this entails the presence of fluctuations in observables determined from event-byevent measurements. These fluctuations may substantially complicate selection of event classes and lead to biased numerical estimates for the mean values of global variables. The accuracy in measurements of observables and the choice of centralityclass width are of crucial importance for correlation experiments aimed at searches for physics effects whose manifestations may be associated with various fluctuations.

Part of fluctuations of observables may be background fluctuations (that is, trivial ones associated with class-selection methods used). Some other ones are so-called volume fluctuations that are present even at fixed impact parameters of collisions because of fluctuations of the initial configurations of nucleons in nuclei and fluctuations of the interaction cross sections. Finally, of greatest interest are dynamical and quantum fluctuations and correlations, which may be of a physical origin-for example, serve as an important signal of QGP formation in scanning the energy region of the possible phase transition. For example, it is natural to expect that, at the critical point of strongly interacting matter on the phase diagram, fluctuations of the multiplicity and mean transverse momentum and their event-by-event correlations are large [19-22].

The first searches for the critical point of strongly interacting matter were performed in the NA49 and NA61/SHINE experiments at CERN $[23,24]$. These investigations have been continued to date by varying both the energy and the masses of colliding nuclei [25]. In particular, the Beam Energy Scan (BES) program was launched for the RHIC nuclear beam in Berkeley [26, 27]. A more detailed analysis of special features of the phase diagram of strongly interacting matter and properties of QCD in the region of a significant baryon excess will become possible after the accumulation of high-statistics data in the future experiments CBM at the FAIR collider (Germany), which is under construction, and MPD at the NICA collider of the Joint Institute of Nuclear Research (JINR, Dubna) [28, 29].

Searches for critical fluctuations of observables involve difficulties stemming from the intricacy of evolution of nucleus-nucleus collisions and from substantial uncertainties in its theoretical description [30]. In particular, perturbative QCD cannot be applied directly in this region.

It is obvious that, in order to interpret correctly experimental data on nucleus-nucleus and protonnucleus collisions, the event-selection procedure used should be constructed in such a way as to minimize fluctuations that appear to be a background to the sought signal and which stem from the scatter of events in the impact parameter and/or in the number of participant nucleons. A detailed analysis of problems encountered in determining centrality classes for $p \mathrm{~Pb}$ collisions at the c.m. energy of $\sqrt{s_{N N}}=5.02 \mathrm{TeV}$ can be found in [10]. In order to understand and analyze the initial-state effect in studying fluctuations and correlations in hadron collisions via experiments conducted at CERN, it is important to solve the problem of this minimization.

The ensuing exposition is organized as follows.

In Section 2, we introduce necessary definitions used in this article below and, within a Monte Carlo simulation, describe thereupon a method for numerically estimating centrality of collisions. This method 
makes it possible to choose centrality classes in such a way that background (volume) fluctuations associated with the dispersion of the impact parameter or the number of participant nucleons $\left(N_{\text {part }}\right)$ are minimized. In this section, we address the problem of obtaining the highest resolution in fixing centrality of nucleus-nucleus and proton-nucleus collisions in terms of $N_{\text {part }}$.

In Section 3, we describe some special features of event generators used in the present study to perform numerical estimations. The results of a simulation and of an optimization of centrality-class selection, along with the widths of these classes, are presented for nucleus-nucleus and proton-nucleus collisions at the LHC energies of 2.76 and $5.02 \mathrm{TeV}$, respectively, as well as for collisions of light nuclei $\left({ }^{7} \mathrm{Be}-\right.$ ${ }^{9} \mathrm{Be}$ ) at the SPS energies.

In Section 4, we analyze problems encountered in estimating centrality in proton-nucleus collisions at the LHC energies. The effect of gluon shadowing on multiparticle-production processes and on the description of the rapidity distribution is studied with the aid of the HIJING 1.38 Monte Carlo event generator. Specifically, we analyze the distribution of events with respect to the number of participant nucleons. This approach is tested on the basis of the non-Glauber Monte Carlo model involving string fusion [31, 32] and on the basis of the modified Glauber model proposed in $[33,34]$. The results are compared with available experimental data.

In the last section, we draw conclusions on the applicability of Glauber scaling in the regions of soft and hard processes and on the observed distinctions.

\section{CLASSES OF CENTRALITY OF NUCLEUS-NUCLEUS COLLISIONS AND VOLUME FLUCTUATIONS \\ 2.1. Centrality}

As a purely geometric notion, a collision centrality implies a specific value of the impact parameter $b$ for each event and the respective value of the nuclear-thickness function. As a spectacular interpretation of the centrality concept, we will consider proton-nucleus collisions. In that case, integration of the nuclear-overlap function $T_{p \mathrm{~Pb}}(b)$ over the whole region of values of the impact parameter $b$ yields the total (geometric) interaction cross section in the form [35]

$$
\sigma_{p \mathrm{~Pb}}=\int d^{2} b \sigma_{\text {inel }}^{p p} T_{p \mathrm{~Pb}}(b),
$$

where $\sigma_{\text {inel }}^{p p}$ is the cross section for inelastic pp interaction. For some chosen collision-centrality class specified by preset upper and lower boundaries of impact-parameter values, $b_{1}$ and $b_{2}$, the cross section assumes the form

$$
\sigma_{p \mathrm{~Pb}}^{b_{1}, b_{2}}=\int_{b_{1}}^{b_{2}} d^{2} b \sigma_{\text {inel }}^{p p} T_{p \mathrm{~Pb}}(b) .
$$

For a specific collision-centrality class, one can determine the fraction of the total geometric cross section (2) as

$$
C^{b_{1}, b_{2}}=\frac{\int_{b_{1}}^{b_{2}} d^{2} b \sigma_{\text {inel }}^{p p} T_{p \mathrm{~Pb}}(b)}{\int d^{2} b \sigma_{\text {inel }}^{p p} T_{p \mathrm{~Pb}}(b)} .
$$

The quantity $C^{b_{1}, b_{2}}$ is frequently used as an eventcentrality measure. Values of the function $C^{b_{1}, b_{2}}$ lie in the following range: $C^{b_{1}, b_{2}} \in[0,1]$. Values close to zero, $C^{0, b_{2}} \sim 0$, correspond to the most central collisions (for which $b_{1}=0$ and $b_{2} \sim 0$ ). For the case of minimum-bias collisions ( $b_{1}=0$ and $b_{2}=\infty$ ), we have $C^{b_{1}, b_{2}} \sim 1$.

However, only indirect estimations of $b$ and $\left\langle N_{\text {coll }}\right\rangle$ are possible in experiments; on the basis of the resulting estimates, one usually selects events and then associates them with one centrality class or another. In a data analysis, event classes chosen on the basis of distributions of some observable are therefore employed instead of values of expression (4). For example, use is extensively made of the distribution of events with respect to the multiplicity of charged particles, $F\left(N_{\mathrm{ch}}\right)$. An event class is specified in terms of respective fractions in just the same way as in expression (4). For some threshold multiplicity value $N_{1}$, it is possible to obtain the respective fraction where $N_{\text {ch }}<N_{1}$ in percentiles; that is,

$$
C_{\exp }^{0, N_{1}}=\frac{\int_{0}^{N_{1}} d z F(z)}{\int d z F(z)} .
$$

For $N_{2}$, we accordingly have

$$
C_{\exp }^{0, N_{2}}=\frac{\int_{0}^{N_{2}} d z F(z)}{\int d z F(z)} .
$$

For some multiplicity-class sample $\left(N_{1}<N_{\text {ch }}<\right.$ $N_{2}$ ), the fraction of events can be obtained in the form

$$
C_{\exp }^{N_{1}, N_{2}}=\frac{\int_{N_{1}}^{N_{2}} d z F(z)}{\int d z F(z)} .
$$

The value of $C_{\mathrm{exp}}^{N_{1}, N_{2}} \in[0,1]$ is usually expressed in percent. It corresponds numerically to the width 
of the chosen multiplicity class. The values of $C_{\text {exp }}^{N_{1}, \infty} \sim 0$ at large $N_{1}$ correspond to the most central collisions.

\subsection{Fluctuations and Correlations}

All quantities in expressions (1) and (2) $\left(N_{\mathrm{ch}}^{p \mathrm{~Pb}}\right.$, $N_{\text {part }}, N_{\text {coll }}$, and $\left.T_{p \mathrm{~Pb}}\right)$-we denote them by $x-$ fluctuate from one event to another. The relative fluctuations of any of these quantities can be estimated as

$$
\omega_{x}=\sigma_{x}^{2} /\langle x\rangle,
$$

where $\sigma_{x}^{2}$ is the variance of an observable and $\langle x\rangle$ is the mean value.

Dynamical fluctuations of the number of multiparticle-production sources may lead to the appearance of so-called long-range correlations [36, 37], which stem from the appearance of sources of a new type because of collective effects [38]. The coefficient $b_{\text {corr }}$ characterizing long-range multiplicity correlations, which is measured on the basis of the number of events in separated rapidity intervals usually referred to as the forward $(F)$ and backward $(B)$ rapidity intervals, is one such characteristic. The coefficient $b_{\text {corr }}$ can be defined as [39]

$$
b_{\mathrm{corr}}=\frac{\left\langle n_{F} n_{B}\right\rangle-\left\langle n_{F}\right\rangle\left\langle n_{B}\right\rangle}{\left\langle n_{F}^{2}\right\rangle-\left\langle n_{F}\right\rangle^{2}},
$$

where $\left\langle n_{F}\right\rangle$ and $\left\langle n_{B}\right\rangle$ are the mean (in some event class) charged-particle multiplicities measured in the $F$ and $B$ intervals.

From Eq. (9), one can see that the quantities on which the correlation coefficient $b_{\text {corr }}$ depends include the value chosen for the width of the centrality class over which averaging occurs. The disregard of this factor may lead to biased physical results [40-45]. Obviously, it is therefore necessary to minimize the role of volume fluctuations and to assess their contribution to measured quantities.

\subsection{Glauber Model and Two-Component Model in Estimations of Collision Centrality}

The nuclear-collision centrality (which is characterized by the impact-parameter value) and the respective number of nucleon-nucleon collisions, $N_{\text {coll }}$, cannot be measured directly in experiments in each individual event. Therefore, these quantities and their average values are strongly model-dependent. At the same time, one can in principle determine the number $N_{\text {part }}$ of participant nucleons by detecting, in a given event, the number $N_{\text {spect }}$ of spectator nucleons-that is, those that did not suffer any inelastic collision. Nevertheless, the Glauber model [46] is widely used to estimate the mean values for both quantities (for an example of implementation, the interested reader is referred to $[47,48]$ ), including $\left\langle N_{\text {coll }}\right\rangle$. In this approach, the respective mean values are determined for a chosen class of nucleus-nucleus collisions on the basis of a fit to multiplicity distributions.

It should be noted that the concept of participant nucleons was introduced in [49], where the mean values and variances of multiplicity distributions in nucleus-nucleus collisions were calculated under the assumption that an inelastic collision of two nuclei is a superposition of incoherent collisions of individual nucleons. It was also assumed in [49] that the mean multiplicity is proportional to the number of so-called wounded nucleons - that is, nucleons that suffered at least one inelastic collision. Later on, it became common practice to refer to such nucleons as participant nucleons.

The standard Glauber model [46] for nucleusnucleus collisions is based on the concept of independent nucleon-nucleon interactions treated in the eikonal approximation and under the assumption of constant $p p$ - and $p \bar{p}$-interaction cross sections, which are taken from experimental data.

In order to describe the distribution of nucleons within a nucleus, one usually employs the wellknown nuclear-matter density distribution for heavy nuclei in the Woods-Saxon form

$$
\rho(r)=\rho_{0}\left\{1+\exp \left(\frac{r-R_{A}}{a}\right)\right\}^{-1} .
$$

The radius of the nucleus $R_{A}$ and the parameter $a$ are determined below from experimental data [50]:

$$
\begin{gathered}
R_{A}=R_{0} \cdot A^{\frac{1}{3}}, \quad \rho_{0}=1.07 \mathrm{fm}, \\
a=0.545 \mathrm{fm} .
\end{gathered}
$$

In the case of light nuclei, the nuclear-matter density is represented in a form that is similar to that for a harmonic oscillator; that is,

$$
\begin{gathered}
\rho(r)=\frac{4}{\pi^{\frac{3}{2}} r_{0}^{3}}\left[1+\frac{A-4}{6}\left(\frac{r}{r_{0}}\right)^{2}\right] \\
\times \exp \left(-\frac{r^{2}}{r_{0}^{2}}\right) .
\end{gathered}
$$

Here, the quantity $r_{0}$ is related to the charge radius $\left(\left\langle r_{\mathrm{ch}}^{2}\right\rangle_{A}\right)$ of the nucleus with mass number $\mathrm{A}$ and the proton charge radius $\left(\left\langle r_{\mathrm{ch}}^{2}\right\rangle_{p}\right)$ by the equation

$$
r_{0}^{2}=\left(\frac{5}{2}-\frac{4}{A}\right)^{-1}\left(\left\langle r_{\mathrm{ch}}^{2}\right\rangle_{A}-\left\langle r_{\mathrm{ch}}^{2}\right\rangle_{p}\right) .
$$


The mean number $N_{\text {part }}(n)$ of participant nucleons in a nucleus-nucleus collision with a multiplicity $n$ can be determined as [51]

$$
\begin{gathered}
\left\langle N_{\text {part }}(n)\right\rangle \\
=\frac{\int d^{2} b P(n ; b)\left(1-P_{0}(b)\right) N_{\text {part }}(b)}{\int d^{2} b P(n ; b)\left(1-P_{0}(b)\right)},
\end{gathered}
$$

where $n(b)$ is the mean multiplicity of charged particles produced in collision of nuclei at a given value of the impact parameter $b$ according to an estimation similar to that based on expression (16); $\left\langle N_{\text {part }}(n)\right\rangle$ is the mean value of participant nucleons within the Glauber approach; $P(n ; b)$ is the correlation function for $n(b)$ (for more details, see [51]); and $P_{0}(b)$ is the probability for the absence of interaction between nuclei at a given value of the impact parameter $b$,

$$
P_{0}(b)=\left(1-\sigma_{\text {inel }}^{p p} T_{A A}(b)\right)^{A^{2}} .
$$

Here, $T_{A A}(b)$ is the nuclear-overlap function for two nuclei of mass number $\mathrm{A}$.

An expression for the number of nucleon-nucleon collisions, $N_{\text {coll }}(n)$, can be obtained [51] by a method similar to that used to derive Eq. (4).

The Glauber model is usually applied together with the so-called two-component model [51], which is based on the assumption that the multiplicities in hard and soft processes are proportional in the case of nuclear collisions both to the number of nucleonnucleon collisions $\left(N_{\text {coll }}\right)$ and to the number of participant nucleons $\left(N_{\text {part }}\right)$. As a result, we arrive at

$$
d N / d y=f n_{p p} N_{\text {part }}+(1-f) n_{p p} N_{\text {coll }},
$$

where $n_{p p}$ is the mean multiplicity of charged particles per pseudorapidity unit (pseudorapidity density) according to measurements in proton-proton and proton-antiproton collisions at a given value of the energy $\sqrt{s}$. The adjustable parameter $f$ is determined in constructing a fit to multiplicity distributions for the case of minimum-bias events. The parameter $f$ may be different for different colliding systems and different energies. Thus, one assumes that the fitting procedure in question, which employs expression (16), makes it possible to obtain numerical values of $N_{\text {coll }}$ that correspond to a specific collisioncentrality class.

For the case of non-single diffractive (NSD) $p p$ and $p \bar{p}$ interactions, use is usually made of the following parametrization of the quantity $n_{p p}$ as a function of $\sqrt{s}$ [52]:

$$
n_{p p}(s)=2.5-0.25 \ln (s)+0.023 \ln ^{2}(s) .
$$

As an interpretation of the two-component model, it is assumed in [38] that the number of charged particles produced in each event of collisions of two particles at an impact parameter $b$ depends exclusively on the number of product single-type sources (quark-gluon strings) $-N_{\text {str }}$ - and is proportional to the source intensity - that is, to the mean number of charged particles per rapidity unit, $m_{n n}=1.1$, that are produced by the source. Here, one employs the Poisson distribution for the multiplicity of particles from one string, and this makes it possible to simulate the ultimate fluctuations of observables.

In the general case of nucleus-nucleus collisions, the impact-parameter $(b)$ dependence of the number of quark-gluon strings produced in an event, $N_{\mathrm{str}}$, has the form

$$
N_{\mathrm{str}}(b)=f N_{\mathrm{str}}^{N N} N_{\text {coll }}(b)+(1-f) N_{\text {part }}(b),
$$

where $N_{\text {coll }}(b)$ is the total number of nucleonnucleon collisions, $N_{\text {part }}(b)$ is the total number of participant nucleons, and $f \in[0,1]$ is an adjustable parameter that depends on the interaction energy.

The dependence of the parameter $f$ on the c.m. nucleon-nucleon collision energy $\sqrt{s_{N N}}$ was obtained earlier [38] in the form

$$
f=0.1731 \ln \left(\sqrt{s_{N N}}\right)-0.4839,
$$

where $N_{\mathrm{str}}^{N N}$ is the number of strings in a nucleonnucleon collision [38]:

$$
\begin{aligned}
N_{\mathrm{str}}^{N N} & =2.56-0.478 \ln \left(\sqrt{s_{N N}}\right) \\
& +0.084\left(\ln \left(\sqrt{s_{N N}}\right)\right)^{2} .
\end{aligned}
$$

In order to study the role of volume fluctuations in an analysis of centrality classes, our group developed a Monte Carlo event generator [53] based on the assumptions outlined above. The results obtained with the aid of this generator agree well with the results of theoretical calculations from [35] and were published earlier in [54].

\subsection{Event-Selection Method Intended for Minimizing Background Fluctuations}

Calculations based on the Monte Carlo generator developed in [53] were performed in order to analyze the effect of fluctuations in processes of chargedparticle production on the mean number of participant nucleons, $\left\langle N_{\text {part }}\right\rangle$, and on its variance, which depend not only on the mean value that specifies a centrality class but also on its width.

It is obvious that, as the width of any chosen centrality class becomes smaller, the variance of $\left\langle N_{\text {part }}\right\rangle$ due to trivial background fluctuations should decrease in general. Concurrently, one expects motion toward a plateau of volume fluctuations associated with fluctuations of cross sections for inelastic nucleon-nucleon interaction. We have already indicated that, even in an ideal case of collisions between 
nuclei at a fixed impact parameter, such fluctuations will inevitably be present in going over from one event to another.

In order to choose an optimum value for the centrality-class width in the minimum-bias distributions of events, simulated distributions with respect to the impact parameter $b$ and with respect to some other observable (multiplicity or number of participant nucleons) were used in the present study.

The results of the Monte Carlo simulation for the minimum-bias $\mathrm{PbPb}$ collisions at $\sqrt{s_{N N}}=2.76 \mathrm{TeV}$ are given in Fig. 1. In Fig. 1a, the vertical lines indicate examples of centrality classes chosen in multiplicity. The distributions with respect to the number of participant nucleons, $N_{\text {part }}$, for these multiplicity classes are presented in Fig. $1 b$. The respective calculations were performed for various centrality and class-width values. The resulting mean values $\left\langle N_{\text {part }}\right\rangle$ and their variances are shown in Figs. $1 c$ and $1 d$.

One can readily notice that, in choosing event classes in the multiplicity $\left\langle N_{\mathrm{ch}}\right\rangle$ of charged particles, the initial distributions with respect to the number of participant nucleons, $\left\langle N_{\text {part }}\right\rangle$, proves to be rather broad even in the case of choosing a multiplicity class of width as small as $1 \%$ (see Fig. 1b). Fluctuations of multiparticle-production processes play a significant role. From Fig. 1c, it follows that, as the width of the centrality class grows, the mean value $\left\langle N_{\text {part }}\right\rangle$ undergoes a systematic bias, which may reach $10 \%$ in the case of the most central collisions.

The calculated values of the variance $\sigma\left(N_{\text {part }}\right)$ in Fig. $1 d$ may serve as a basis for an optimum choice of multiplicity-class width. From the calculated results shown in this figure, one can clearly see that, for central and semiperipheral collisions, trivial fluctuations decrease substantially as the width of the multiplicity class becomes smaller. At the same time, the choice of narrow intervals for multiplicity classes becomes meaningless for peripheral collisions, since, in this case, the contribution of volume fluctuations remains relatively large.

Thus, a minimization of volume fluctuations is possible for central collisions via the reduction of the centrality-class width. The level of volume fluctuations in the region where a plateau is presumably reached and the optimum width of the centrality class may be discussed and used in the physics analysis.

\subsection{Collisions of Light Nuclei: ${ }^{7} \mathrm{Be}-{ }^{9} \mathrm{Be}, 16.8 \mathrm{GeV}$}

An analogous analysis on the basis of the Glauber model with the Monte Carlo event generator from [53] was also performed for collisions of ${ }^{7} \mathrm{Be}-{ }^{9} \mathrm{Be}$ light nuclei at the c.m. energy of $\sqrt{s_{N N}}=16.8 \mathrm{GeV}$. Qualitatively, the results of this analysis are similar to those that were presented above. For illustrative purposes, they are presented in Fig. 2. Irrespective of the choice of multiplicity intervals (their boundaries and width), the variance $\sigma\left(N_{\text {part }}\right)$ is so large that it is impossible to use effectively the concept of a multiplicity class as a criterion for the choice of centrality in the case of collisions of light nuclei (see Fig. $2 b$ ). It is obvious that, both for peripheral and for central collisions, an event selection in multiplicity would lead in this case to the mixing of events featuring a high level of fluctuations of $N_{\text {part }}$.

\section{FLUCTUATIONS AND THE NUMBER OF PARTICIPANT NUCLEONS IN $p \mathrm{~Pb}$ COLLISIONS}

Similar calculations were also performed for the case of $p \mathrm{~Pb}$ collisions at $\sqrt{s_{N N}}=5.02 \mathrm{TeV}$. From the results of the calculations performed on the basis of the Glauber model with the Monte Carlo event generator from [53], it follows that, in the case of $p \mathrm{~Pb}$ collisions, a decrease in the number of participant nucleons is accompanied by a substantial growth of fluctuations of multiparticle production. Those calculations also show that it becomes impossible to establish a clear-cut correspondence between centrality and events in which the number $N_{\text {part }}$ of participant nucleons have a preset fixed value.

The effect of initial conditions on the mean values of observables and on the magnitude of their fluctuations is also confirmed by the results of a numerical simulation performed for minimum-bias $p \mathrm{~Pb}$ collisions at the c.m. energy of $\sqrt{s_{N N}}=5.02 \mathrm{TeV}$ with the aid of the HIJING 1.38 event generator [55, 56] (there is a free access to the last version of this generator).

\subsection{Fluctuations and Number of Participant Nucleons in the HIJING 1.38 Event Generator}

In contrast to the Glauber model, the HIJING 1.38 event generator involves collective internal effects of the interaction of nuclei-for example, it takes into account an in-medium modification of the parton distribution. The experiments reported in [5759 ] revealed a difference between the parton distributions in nuclei and in the proton, and this leads to a decrease in the cross section at small $x$. In the calculations, this can be controlled by varying the gluon-shadowing parameter $s_{g}$, which takes values in the range of $s_{g} \in[0.0 ; 1.0]$. It is determined from a fit to experimental rapidity distributions. At the value of $s_{g}=0$, all nucleon-nucleon interactions are 

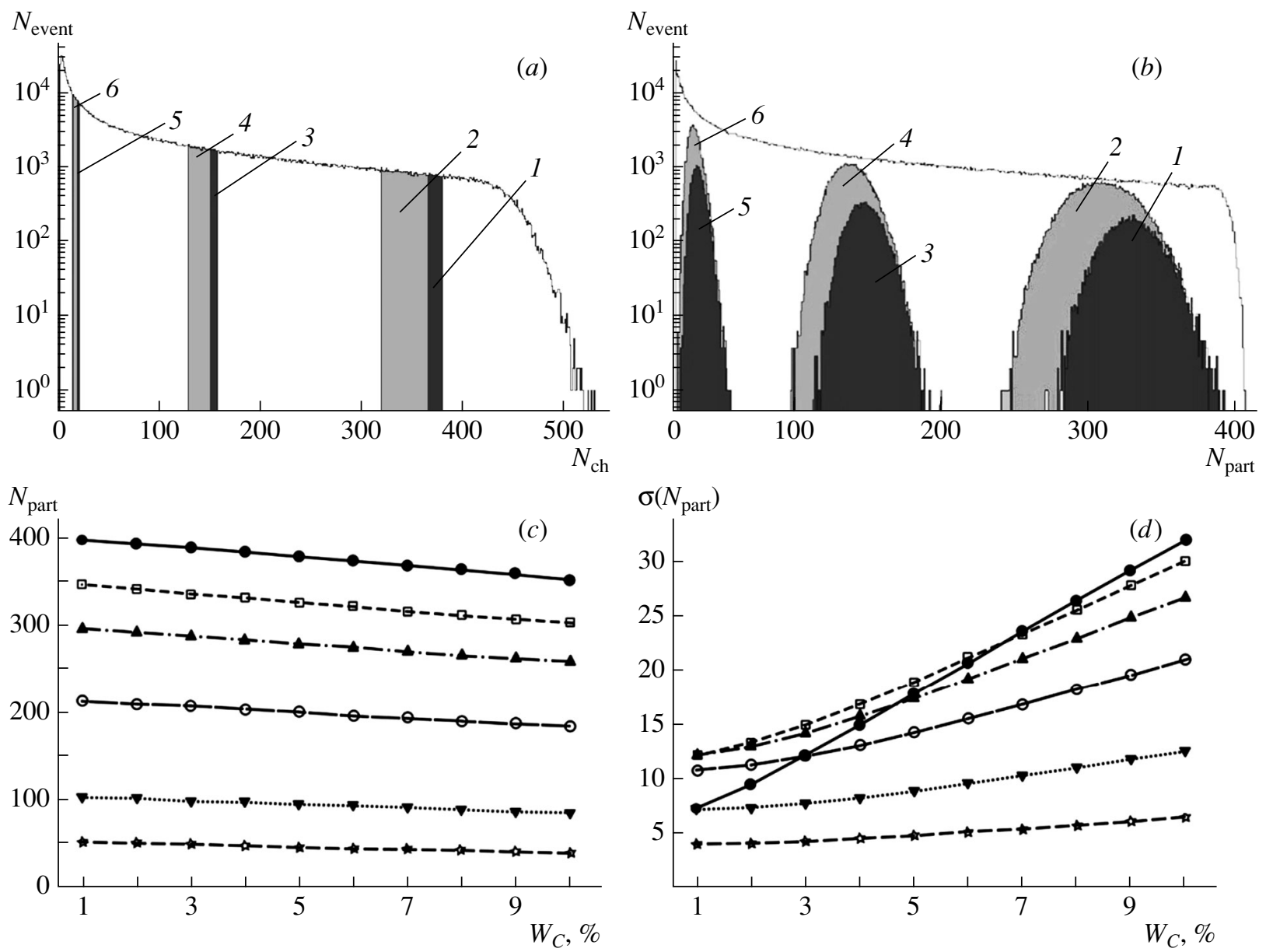

Fig. 1. Distribution of the number of events $(N)$ for $\mathrm{PbPb}$ collisions at the c.m. energy of $\sqrt{s}=2.76 \mathrm{TeV}$ (calculation on the basis of the Glauber model with the Monte Carlo event generator from [53]) with respect to the (a) multiplicity $N_{\text {ch }}$ and ( $b$ ) number $N_{\text {part }}$ of participant nucleons for the following chosen classes of multiplicity and centrality $C$ : (1) $5-6 \%,(2) 5-10 \%,(3)$ $30-31 \%$, (4) 30-35\%, (5) $70-71 \%$, and (6) $70-75 \%$; analogous distributions of (c) the mean number of participant nucleons ( $\left.N_{\text {part }}\right)$ and $(d) \sigma\left(N_{\text {part }}\right)$ for various classes of $N_{\text {ch }}$ versus their widths $W_{\mathrm{C}}$. The points stand for the lower boundaries of the centrality classes $C: C=$ (closed circles) $0 \%$, (open boxes) $5 \%$, (closed triangles) $10 \%$, (open circles) $20 \%$, (inverted closed triangles) $40 \%$, and (stars) $60 \%$. In just the same way as in Fig. 4, the curves are drawn to guide the eye.
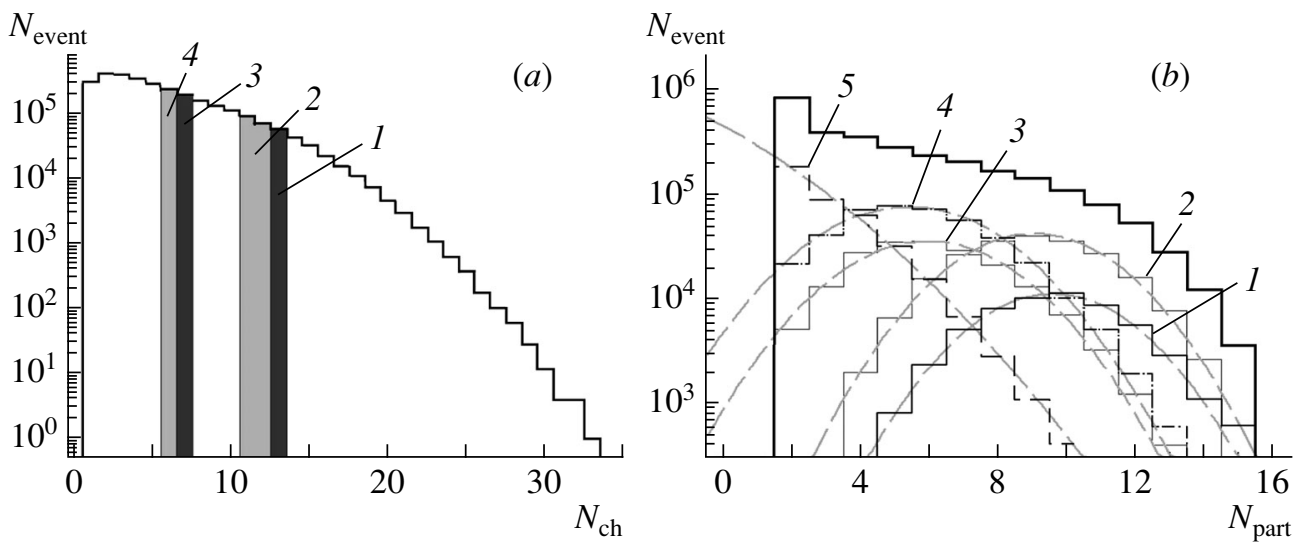

Fig. 2. As in Figs. $1 a$ and $1 b$, but for ${ }^{7} \mathrm{Be}-{ }^{9}$ Be collisions at the energy of $\sqrt{s}=16.8 \mathrm{GeV}$; in Fig. $2 b$, the approximating curves has a Gaussian form. 

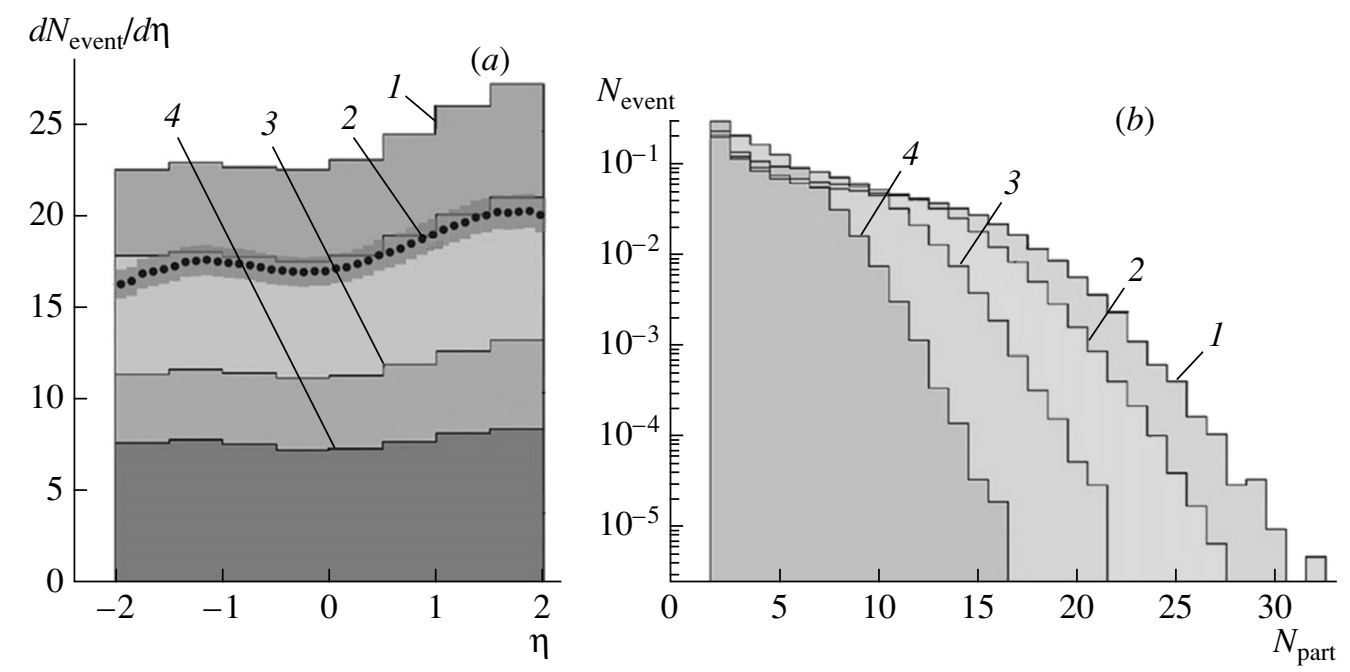

Fig. 3. Results for $p \mathrm{~Pb}$ collisions at the energy of $\sqrt{s_{N N}}=5.02 \mathrm{TeV}$ according to calculations performed with the aid of the HIJING 1.38 event generator: $(a)$ rapidity $(\eta)$ distributions of charged particles (the displayed points stand for experimental data from [9]) and $(b)$ distributions of $N$ with respect to the number $N_{\text {part }}$ of participant nucleons for various values of the gluon-shadowing parameter $s_{g}$ (histograms): (1) 0.0 , (2) $0.1,(3) 0.2$, and (4) 0.28 .

assumed to be independent (in just the same way as in the Glauber model).

We have analyzed the influence of the collective gluon-shadowing effect on the mean value and variance of $N_{\text {part }}$ for $p \mathrm{~Pb}$ collisions. The results of various versions of HIJING 1.38 calculations for $p \mathrm{~Pb}$ collisions at $5.02 \mathrm{TeV}$ are given in Fig. 3 for several values of the parameter $s_{g}$. Figure $3 a$ shows that the rapidity distribution of the charged-particle multiplicity depends greatly on $s_{g}$. The calculations leading to the results presented in this figure along with experimental data reported in [9] and obtained by the ALICE Collaboration in the pseudorapidity range of $\{-2,2\}$ (points in Fig. $3 a$ ) make it possible to choose the parameter value of $s_{g}=0.1$ for the HIJING 1.38 version.

Figure $3 b$ shows that the distribution of the number of events with respect to the number $N_{\text {part }}$ of participant nucleons changes strongly with the gluonshadowing parameter $s_{g}$.

Both the mean values $\left\langle N_{\text {part }}\right\rangle$ for minimum-bias events and the variance $\sigma\left(N_{\text {part }}\right)$ change substantially.

A decrease in the mean value $\left\langle N_{\text {part }}\right\rangle$ for $p \mathrm{~Pb}$ collisions at the energy of $5.02 \mathrm{TeV}$ upon the inclusion of the gluon-shadowing effect is an important consequence of our present calculations.

\subsection{Non-Glauber Model of Nucleus-Nucleus Collisions}

In the present study, we also use a non-Glauber Monte Carlo model that takes into account collective initial-state effects in the form of the fusion of quarkgluon stings and which relies on the parton picture of nucleon-nucleon interaction [31, 32, 60]. The conceptual framework of the model also includes the following assumptions:

(i) Each parton can undergo only one interaction with another parton (in contrast to Glauber's assumption of sequential nucleon-nucleon collisions in which the cross sections for inelastic nucleon interaction is constant).

(ii) A nucleon from one nucleus takes part in a collision if at least one of its partons collides with a nucleon from the collision partner of this nucleus.

(iii) Data on the multiplicity and on the total inelastic cross section for proton-proton interaction determine (constrain) model parameters.

(iv) A consistent description of multiplicities in the minimum-bias rapidity distributions in $p \mathrm{~Pb}$ collisions is yet an additional requirement.

For $\mathrm{PbPb}$ collisions at the $\mathrm{c}$.m. energy of $\sqrt{s_{N N}}=$ $2.76 \mathrm{TeV}$, Fig. 4 demonstrates the role of fluctuations of the initial distributions of parton configurations in nuclei and the role of the production, fusion, and hadronization of quark-gluon strings as independent sources of the multiparticle production of charged particles in the soft and semihard regions of the transverse-momentum spectrum. This figure shows how the coefficient $b_{\text {corr }}$ characterizing longrange multiplicity correlations changes for various values of the collision centrality versus the width of the centrality class. These results demonstrate that $b_{\text {corr }}$ decreases substantially upon the shrinkage 


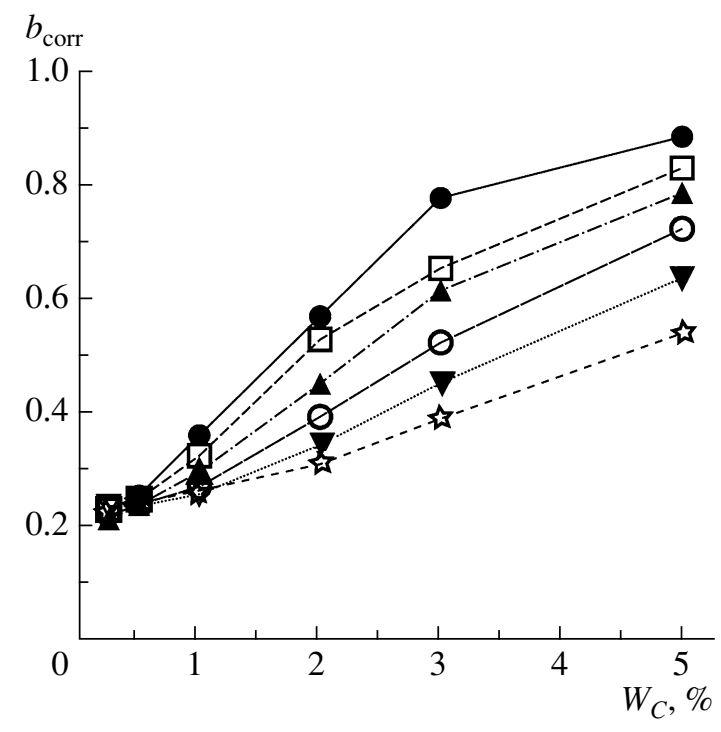

Fig. 4. Multiplicity-correlation coefficient $b_{\text {corr }}$ calculated within the non-Glauber approach for $\mathrm{PbPb}$ collisions at the c.m. energy of $\sqrt{s}=2.76 \mathrm{TeV}$ for various multiplicity classes $N_{\text {ch }}$ versus their widths $W_{\mathrm{C}}$. The notation for the centrality $(C)$ lines is identical to that in Figs. $1 c$ and $1 d$.

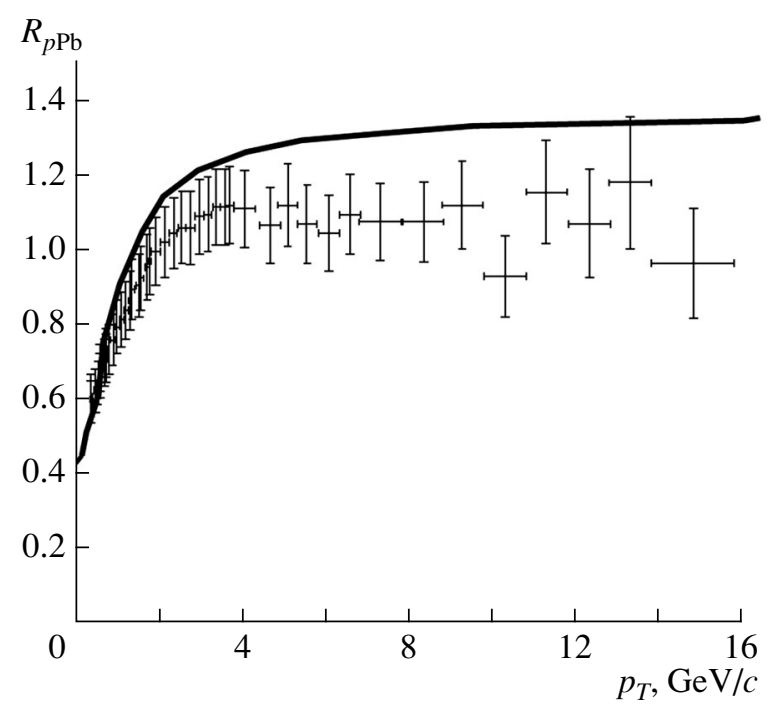

Fig. 5. Nuclear-modification factor $R_{p \mathrm{~Pb}}$ as a function of the transverse momentum $p_{T}$. The curve represents the results of the calculations based on the non-Glauber approach [31]. The displayed points stand for experimental data from [6].

of the centrality class and reaches a constant value (plateau), the growth of $b_{\text {corr }}$ in broader classes upon a transition from peripheral to central collisions disappearing.

The transverse-momentum $\left(p_{T}\right)$ dependence of the nuclear-modification factor $R_{p \mathrm{~Pb}}$ calculated on the basis of the non-Glauber model with allowance for collective initial-state effects in the form of interacting (merging together) quark-gluon strings for the case of $p \mathrm{~Pb}$ collisions is shown in Fig. 5 along with experimental data from [6]. From this figure, one can see that the model proposed in $[31,32$, $60]$, which describes correctly the multiplicity in the minimum-bias rapidity distributions in $p \mathrm{~Pb}$ collisions and which determines correctly the initial conditions for nucleus-nucleus collisions, provides an accurate qualitative description of the behavior of the nuclearmodification factor as a function of $p_{T}$ both in the soft and in the hard region of the transverse-momentum spectrum. The fact that the value of $R_{p \mathrm{~Pb}}$ in the soft region is substantially smaller than unity is due to energy conservation in elementary nucleon-nucleon collisions [61, 62].

\subsection{Modified Glauber Model and Number of Nucleon-Nucleon Collisions}

It has been shown above that, if the multiplicity is used as a criterion in choosing classes of event centrality, biased estimates can be caused by fluctuations of the multiparticle production of charged particles. Moreover, initial-state effects, such as shadowing (within the HIJING scheme) and the fusion of quark-gluon strings, where the law of energy-momentum conservation holds, lead to substantially smaller mean values of the number of nucleon-nucleon collisions than those that arise in the standard Glauber model. This is because expression (14) was obtained under the assumption that all consecutive nucleon-nucleon collisions occur independently at the same energy $\sqrt{s_{N N}}$ and have the same cross section $\sigma_{N N}$. However, it can be stated that these assumptions are oversimplified. The point is that both the energy of successive elementary inelastic nucleon collisions and the respective value of the cross section $\sigma_{N N}$ may change. In particular, multiparticle production processes and the energy loss in the dominant region of soft processes should lead to a momentum loss and to a change in the value of $\sigma_{N N}$ for subsequent collisions and may accordingly change the values of $N_{\text {part }}$ and $N_{\text {coll }}$. Thus, expression (14) becomes incorrect, and it is natural to assume that, within any approach that disregards the energy loss by particle production, the values of $N_{\text {coll }}$ will prove to be exaggerated.

Earlier, momentum-conservation effects were taken into account on the basis of the modified Glauber model (MGM) proposed in [33]. Later on, yet another MGM modification that is harnessed in the present study and in which the effects of a repulsive core and secondary nucleon rescattering are taken additionally into account was developed 
Mean number of participant nucleons, $N_{\text {part }}$ in minimumbias events of $p \mathrm{~Pb}$ collisions at the energy of $5.02 \mathrm{TeV}$ within various models

\begin{tabular}{c|l|c|c}
\hline & Model & $\left\langle N_{\text {part }}\right\rangle$ & References \\
\hline 1 & Glauber & $7.9 \pm 0.6$ & {$[9]$} \\
2 & MGM & $4.3 \pm 0.3$ & {$[61]$} \\
3 & Non-Glauber & $6.2 \pm 0.6$ & {$[62]$} \\
4 & HIJING & 6.5 & {$[53]$} \\
5 & AMPT & 6.4 & {$[18]$} \\
\hline
\end{tabular}

in $[34,61]$. This model is similar to the Glauber model but is employed to describe the dependence of the total multiplicity of charged particles in nucleusnucleus collisions on $\sqrt{s_{N N}}$ with one adjustable parameter, $k=0.35$, which determines the nucleontransverse-momentum fraction that remains after each inelastic nucleon-nucleon collision and the respective momentum and cross section for the next collision. The MGM approach provides a correct description of the total-multiplicity yield per number of participant nucleons, $N_{\text {part }}$, in the energy range between $17 \mathrm{GeV}$ and $2.76 \mathrm{TeV}$ [61]. At the same time, the model involving the parameter set to $k=0.35$ predicts substantial proton stopping in lead nuclei: the mean value $\left\langle N_{\text {coll }}\right\rangle$ for the minimum-bias $p \mathrm{~Pb}$ collisions at the c.m. collision energy of $\sqrt{s_{N N}}=$ $5.02 \mathrm{TeV}$ proves to be substantially smaller than that in the standard Glauber model.

In the first results [9] of the ALICE collaboration, a value of $16.81 \pm 0.71$ was obtained for the number of charged particles per pseudorapidity unit in the case of NSD events in $p \mathrm{~Pb}$ collisions at the energy of $5.02 \mathrm{TeV}$. After a normalization to the mean number of participant nucleons $\left(\left\langle N_{\text {part }}\right\rangle\right)$, this value became $2.14 \pm 0.17$, which is smaller than the respective value for $p p$ collisions, the latter assuming the value of 2.6 after the interpolation of CMS data from [11] to the energy of $5.02 \mathrm{TeV}$.

The results obtained by calculating $\left\langle N_{\text {part }}\right\rangle$ within various models that take into account conservation laws and some collective initial-state effects and which describe correctly rapidity distributions of charged-particle multiplicities in $p \mathrm{~Pb}$ collisions are given in the table. Within all of these models, the mean number of participant nucleons in $p \mathrm{~Pb}$ collisions is smaller than that within the Glauber model. These results are indicative of the need for revising the application of the standard Glauber model in the soft part of the transverse-momentum spectrum. This is a point of paramount importance since the region of soft transverse momenta makes an overwhelmingly dominant contribution to the total yield of chargedparticle multiplicities.

\section{CONCLUSIONS}

We have developed methods for determining collision centralities on the basis of multiplicity distributions of events. In our specific calculations, we relied on the Glauber approximation, a twocomponent model that employs numerical estimations of fluctuations within the developed Monte Carlo approaches with allowance for the energymomentum conservation law in nucleon-nucleon collisions, the HIJING and AMPT event generators, and a non-Glauber model that takes into account collective initial-state effects in the form of the fusion of quark-gluon strings. The following conclusions can be drawn from the resulting estimates of $N_{\text {part }}$ and $N_{\text {coll }}$ :

(i) In choosing collision-centrality classes on the basis of multiplicity distributions of events, it is possible to optimize the event-selection procedure in such a way as to reduce the effect of trivial initial-state fluctuations, especially in the case of central nucleusnucleus collisions. Nevertheless, a contribution of trivial fluctuations always remains present, and one should take this into account in analyzing dynamical fluctuations and correlations, since the event class selected in this way is a mixture of collisions characterized by different values of the number $N_{\text {part }}$ of participant nucleons. The estimations performed in the present study lead to the following result: for $1 \%$ in the width of the particle-multiplicity class in $\mathrm{PbPb}$ collisions at the c.m. collision energy of $\sqrt{s_{N N}}=$ $2.76 \mathrm{TeV}$, the root-mean-square deviation $\sigma\left(N_{\text {part }}\right)$ may be about 5 to $10 \%$ for central collisions.

(ii) The inclusion of conservation laws, which is performed in all of the approaches other than that which is based on the standard Glauber model, reduces substantially, in relation to this model, the mean number of nucleon collisions, $\left\langle N_{\text {coll }}\right\rangle$, which is used both to normalize the measured yields of charged particles in nucleus-nucleus and proton-nucleus collisions and to determine the nuclear-modification factor.

(iii) Collective initial-state effects within the nonGlauber model that takes into account the fusion of quark-gluon strings, confirm that there are substantial changes in $\left\langle N_{\text {coll }}\right\rangle$ in relation to what we have within the Glauber approach; moreover, the inclusion of these effects enabled us to describe correctly the behavior of the nuclear-modification factor $p_{T}$ in $p \mathrm{~Pb}$ collisions at the LHC energy.

By and large, our results demonstrate that it is necessary to revise the Glauber model, which 
is widely used in normalizing multiplicity yields in experimental data on proton-nucleus and nucleusnucleus collisions in the soft region of the transversemomentum spectrum of charged particles.

\section{ACKNOWLEDGMENTS}

We are grateful to V.V. Vechernin for numerous discussions on the problems considered in the present study and for enlightening comments.

This work was supported by a grant (no. 11.38.193.2014) from St. Petersburg State University (SPSU). V.N. Kovalenko gratefully acknowledges the support of an SPSU scholarship, the Dynasty Foundation, and a G-RISC scholarship.

\section{REFERENCES}

1. J. P. Blaizot, E. Iancu, and A. Rebhan, in Quark Gluon Plasma 3, Ed. by R. C. Hwa and X.-N. Wang (World Sci., Singapore, 2004), p. 60.

2. A. V. Smilga, Phys. Rep. 291, 1 (1997).

3. D. H. Rischke, Prog. Part. Nucl. Phys. 52, 197 (2004).

4. J. L. Albacete, A. Dumitru, and C. Marquet, Int. J. Mod. Phys. A 28, 1340010 (2013).

5. J. L. Albacete and C. Marquet, Prog. Part. Nucl. Phys. 76, 1 (2014).

6. ALICE Collab. (B. B Abelev et al.), Phys. Rev. Lett. 110, 082302 (2013); arXiv: 1210.4520 [nucl-ex].

7. ALICE Collab. (B. Abelev et al.), Phys. Lett. B 728, 25 (2014).

8. ALICE Collab. (B. Abelev et al.), Eur. Phys. J. C 74, 3054 (2014).

9. ALICE Collab. (B. Abelev et al.), Phys. Rev. Lett. 110, 032301 (2013).

10. AliCE Collab. (J. Adam et al.), Phys. Rev. C 91, 064905 (2015).

11. CMS Collab. (V. Khachatryan et al.), Phys. Rev. Lett. 105, 022002 (2010).

12. CMS Collab. (V. Khachatryan et al.), Eur. Phys. J. C 75, 237 (2015).

13. The ATLAS Collab., ATLAS Note, ATLAS-CONF2014-029.http://cds.cern.ch/record/1704978

14. STAR Collab. (J. Adams et al.), Phys. Rev. Lett. 91, 072304 (2003).

15. K. J. Eskola, H. Paukkunen, and C. A. Salgado, J. High Energy Phys. 0904, 065 (2009).

16. L. Frankfurt and M. Strikman, Phys. Rep. 160, 235 (1988).

17. A. Accardi, in Hard Probes in Heavy Ion Collisions at the LHC, CERN-2004-009 (CERN, Geneva, 2004), p. 81.

18. J. L. Albacete et al., Int. J. Mod. Phys. E 22, 1330007 (2013).

PHYSICS OF ATOMIC NUCLEI Vol.79 No. 52016
19. M. Stephanov, K. Rajagopal, and E. Shuryak, Phys. Rev. D 60, 114028 (1999).

20. M. A. Stephanov, Phys. Rev. D 81, 054012 (2010).

21. NA49 Collab. (T. Anticic et al.), PoS (CPOD 2009), 029 (2009).

22. M. Stephanov et al., Phys. Rev. Lett. 81, 4816 (1998).

23. K. Grebieszkow, Acta Phys. Polon. B 41, 427 (2010).

24. K. Grebieszkow (NA49 and NA61/SHINE Collab.), PoS (EPS-HEP 2009), 030 (2009).

25. M. Gazdzicki, M. Gorenstein, and P. Seyboth, Acta Phys. Polon. B 42, 307 (2011).

26. L. Kumar, Mod. Phys. Lett. A 28, 1330033 (2013).

27. STAR Collab. (M. M. Aggarwal et al.), arXiv: 1007.2613v1 [nucl-ex].

28. V. Friese, PoS (CPOD07), 056 (2007).

29. L. Yordanova and V. Vasendina, J. Phys.: Conf. Ser. 503, 012041 (2014).

30. K. Fukushima, J. Phys. G 39, 013101 (2012).

31. V. N. Kovalenko, Phys. At. Nucl. 76, 1189 (2013).

32. V. Kovalenko and V. Vechernin, PoS (Baldin ISHEPP XXI), 077 (2012).

33. G. Feofilov and A. Ivanov, J. Phys.: Conf. Ser. 5, 230 (2005).

34. A. Seryakov and G. Feofilov, PoS (Baldin ISHEPP XXII), 082 (2015).

35. V. V. Vechernin and H. S. Nguyen, Phys. Rev. C 84, 054909 (2011).

36. A. Capella and A. Krzywicki, Phys. Rev. D 18, 4120 (1978).

37. V. Vechernin, Nucl. Phys. A 939, 21 (2015).

38. V. V. Vechernin and R. S. Kolevatov, Phys. At. Nucl. 70, 1797 (2007).

39. ALICE Collab. (J. Adam et al.), J. High Energy Phys. 1505, 097 (2015).

40. STAR Collab. (B. I. Abelev et al.), Phys. Rev. Lett. 103, 172301 (2009).

41. T. Lappi and L. McLerran, Nucl. Phys. A 832, 330 (2010).

42. T. Lappi, Nucl. Phys. A 854, 117 (2011).

43. A. Bzdak and K. Woźniak, Phys. Rev. C 81, 034908 (2010).

44. V. Kovalenko and V. Vechernin, Eur. Phys. J. Web Conf. 66, 04015 (2014).

45. Yu-L. Yan et al., Phys. Rev. C 81, 044914 (2010).

46. R. J. Glauber and G. Matthiae, Nucl. Phys. B 21, 135 (1970).

47. M. L. Miller, K. Reygers, S. J. Sanders, and P. Steinberg, Ann. Rev. Nucl. Part. Sci. 57, 205 (2007).

48. A. S. Galoyan and V. V. Uzhinsky, Phys. Part. Nucl. Lett. 12, 166 (2015).

49. A. Bialas, M. Bleszyński, and W. Czyz, Nucl. Phys. B 111, 461 (1976). 
50. H. de Vries, C. W. de Jager, and C. de Vries, At. Data Nucl. Data Tables 36, 495 (1987).

51. D. Kharzeev and M. Nardi, Phys. Lett. B 507, 121 (2001).

52. F. Abe et al., Phys. Rev. D 41, 2330(R) (1990).

53. T. Drozhzhova, PoS (Baldin ISHEPP XXII), 070 (2015).

54. T. Drozhzhova, in Proceedings of International Student Conference "Science and Progress," St. Petersburg, 2010.

55. X.-N. Wang and M. Gyulassy, Phys. Rev. D 44, 3501 (1991).

56. M. Gyulassy and X.-N. Wang, Comput. Phys. Commun. 83, 307 (1994).
57. F. Arleo and Th. Gousset, Phys. Lett. B 660, 181 (2008).

58. A. Adeluyi and C. A. Bertulani, Phys. Rev. C 85, 044904 (2012).

59. V. Guzey, E. Kryshen, M. Strikman, and M. Zhalov, Phys. Lett. B 726, 290 (2013).

60. V. Kovalenko, PoS (QFTHEP 2013), 052 (2013).

61. T. Drozhzhova, G. Feofilov, V. Kovalenko, and A. Seryakov, PoS (QFTHEP 2013), 053 (2013).

62. V. N. Kovalenko, arXiv: 1308.1932 [hep-ph]. 